\title{
Analysis on the Applicability of a K-value Model Used for Water Vapor Conversion in Western China
}

\author{
Maohua Yao ${ }^{1}$, Wenting Zhou ${ }^{2, *}$, Xiangping Chen ${ }^{1}$ \\ ${ }^{1}$ Natural Resources Information Center of Guangxi Zhuang Autonomous Region, Nanning, Guangxi, 530023, China \\ ${ }^{2}$ Guilin University of Technology at Nanning, Department of Civil and Surveying Engineering, Chongzuo, Guangxi, 530023, China
}

\begin{abstract}
This paper makes use of the sounding data of 43 sounding stations located in western China from 2016 to 2019 and takes the PWV-ZWD (precipitable water vapor and Zenith wet delay) conversion coefficient $\mathrm{K}$ calculated via numerical integration as the reference value to study the applicability of using a K-value model for PWV-ZWD conversion in western China. Six groups of stations with similar latitude and increasing altitude difference were selected to identify how the K-value model varies with elevation. Also, the K-value model was employed to invert the PWV of eight sounding stations in 2019 to verify the usability of the model. As shown by the results, the establishment of the regional model has contributed to the improvement of model accuracy; the K-value model assumes a higher accuracy in flatter terrains; there is a $<3 \mathrm{~mm}$ deviation between the PWV inverted using the $\mathrm{K}$-value model and that provided by the sounding stations, with the deviation being positively correlated with the PWV value inverted using the K-value model.
\end{abstract}

\section{Introduction}

This study uses the conversion coefficient between precipitable water vapor (PWV) and Zenith wet delay (ZWD), $\mathrm{K}$, in the analysis the ground-based GPS inversion of PWV, and takes it as a key parameter in ground-based GPS meteorology research ${ }^{[1,4]}$. In the research on $\mathrm{K}^{[1-6]}$, most scholars have been adopting integral method, Bevis model or regional Tm model to carry out the calculation of $\mathrm{K}$. Nevertheless, given that these methods require the meteorological parameters of a station, such as ground temperature, air pressure, and even water vapor pressure, the parameter acquisition in reality takes up considerable observation costs and manpower, especially in areas with complex terrains, revealing their limitations ${ }^{[4-5]}$. Emardson et al. [2-3] proposed the Emardson model (hereinafter referred to as the K-value model), which only required the measurement station's latitude and DOY rather than meteorological elements. Liu Lilong et al. [4-5] analyzed the applicability of the K-value model in Xinjiang, while Chen Xiangping et al. [6] explored the applicability of inverting PWV through the H-model, which uses the water vapor conversion coefficient in Qinghai-Tibet Plateau. In this paper, the applicability of K-value model was explored based on the sounding data of 43 sounding stations in western China from 2016-2019, with a view to identifying the impact of altitude difference on the Kvalue model and verifying the feasibility of inverting PWV through the K-value model.

\section{Model Establishment and Applicability Analysis}

Based on the sounding data of 43 sounding stations in western China from 2016 to 2019, the K-value model for atmospheric water vapor conversion over a large area was established in this paper, and the western area was divided into three regions according to the distribution of sounding stations. Among them, Region 1 had 12 stations with an elevation between $664 \mathrm{~m}$ and $1770 \mathrm{~m}$, which were located in the northwest border area of China; located in central and western China were 18 stations in Region 2, which covers Tibet, Gansu, Qinghai and Inner Mongolia; located in the lower latitudes of southwest China, Region 3 consisted of 13 stations covering those with relatively flat terrains, such as Guangxi, Yunnan, Guizhou, and Chongqing.

Numerical integration was introduced to carry out the calculation of atmospheric water vapor conversion coefficient from the measured data of sounding stations in western China from 2016 to $2019^{[4]}$. The formula was as follows:

$$
\mathrm{K}=10^{6} / \rho R_{v}\left(k_{3} / T_{m}+k_{2}^{\prime}\right)
$$

where $\rho$ is the density of water, $R_{v}$ is the vapor gas constant, $k_{2}^{\prime}$ and $k_{3}$ are atmospheric refraction constants, and $T_{m}$ is the weighted average temperature.

$$
T_{m}=\frac{\int \frac{P_{v}}{T} d_{z}}{\int \frac{P_{v}}{T^{2}} d_{z}}=\frac{\sum \frac{P_{v i}}{T_{i}} \cdot \Delta h_{i}}{\sum \frac{P_{v i}}{T_{i}^{2}} \Delta h_{i}}
$$

\footnotetext{
$\overline{{ }^{*} \text { Corresponding author: } 136101728 @ q q}$ q.com
} 
where, $P_{v i}$ is the average vapor pressure $(h P a)$ of layer $i ; T_{i}$ is the average temperature $(K)$ of layer $i ; \Delta h_{i}$ is the thickness of layer $i ; P_{v i}, T_{i}$ and $\Delta h_{i}$ can be obtained from sounding station data.

Taking K calculated by Formula (1) as the reference value, the model parameters can be obtained using the least square method to establish the K-value model of the whole area. The model is as expressed in (3).

$K^{-1}=0.16728-0.00033 \varphi-0.00164 \sin \left(2 \pi \frac{d o y}{365}\right)-0.00493 \cos \left(2 \pi \frac{d o y}{365}\right)$

The corresponding K-value models were established for regions 1-3 respectively to calculate the $\mathrm{K}$ for each region. The respective models can be expressed as follows.

$\left\{\begin{array}{l}K_{1}^{-1}=0.16170-0.00016 \varphi-0.00205 \sin \left(2 \pi \frac{d o y}{365}\right)-0.00638 \cos \left(2 \pi \frac{d o y}{365}\right) \\ K_{2}^{-1}=0.14880+0.00014 \varphi-0.00208 \sin \left(2 \pi \frac{d o y}{365}\right)-0.00576 \cos \left(2 \pi \frac{d o y}{365}\right) \\ K_{3}^{-1}=0.16769-0.00028 \varphi-0.00067 \sin \left(2 \pi \frac{d o y}{365}\right)-0.00248 \cos \left(2 \pi \frac{d o y}{365}\right)\end{array}\right.$

The whole-area K-value model derived using Formula (3) was applied to each region, and the application accuracie of regional K1, K2 and K3 models derived using Formula (4) was as shown in Table 1. RMSE measures the mean deviation between the calculated value and the actual value of the model, while MAE offers a measure of reliability.

Table 1. RMSE and MAE of Whole Area K-value Model and Regional K-value Model from 2016 to 2019

\begin{tabular}{ccccc}
\hline Models & $\begin{array}{c}\text { Number } \\
\text { of stations }\end{array}$ & $\begin{array}{c}\text { RMSE } \\
/ 10^{-3}\end{array}$ & $\begin{array}{c}\text { MAE } \\
/ 10^{-3}\end{array}$ \\
\hline $\begin{array}{c}\text { The } \\
\text { regional } \\
\text { models }\end{array}$ & K1 model & 12 & 2.668 & 2.088 \\
\hline \multirow{2}{*}{ The K- } & K2 model & 18 & 3.590 & 2.826 \\
value & Regions 1 & 13 & 2.491 & 1.982 \\
models & Regions 1 & 18 & 3.139 & 2.493 \\
& Regions 1 & 13 & 3.453 & 3.600 \\
& The whole-area & 43 & 3.777 & 2.800 \\
\hline
\end{tabular}

As shown in Table 1, the K-value model based on data of the whole western area demonstrated a low accuracy when applied to all regions, while the regional $\mathrm{K}$-value models based on the distribution of sounding stations assumed favorable accuracy. Among the regional models, the K-value models of Region 1 and Region 3, which are flatter and lower in latitude, assumed better applicability and greater accuracy, whereas the K-value model of Region 2, which has large topographical fluctuation (from the highest altitude at $4508 \mathrm{~m}$ in Naqu Station, Xizang, to the lowest altitude at $411 \mathrm{~m}$ in Jinghe Station) was of lower accuracy. It was therefore concluded that in a large area, dividing the area in accordance the distribution of sounding stations and establishing the regional $\mathrm{K}$-value model can improve the model's accuracy, whereas the accuracy of K-value model is low in regions with a large altitude difference.

\section{Analysis of the Model's Applicability with Respect to Altitude Difference}

In order to identify the impact of altitude difference on K-value model, six groups of stations with similar latitude and increasing altitude difference were selected as the research objects, namely Yinchuan \& Minqin, Pingliang \& Yuzhong, Nanning \& Simao, Yan'an \& Golmud, Hanzhong \& Yushu, Ganzi \& Naqu, with the minimum altitude difference being $255 \mathrm{~m}$ and the maximum being $3986 \mathrm{~m}$. The RMSE and MAE of $\mathrm{K}$ in each station from 2016 to 2019 were calculated using the $\mathrm{K}$-value model derived from Formula (3), as shown in Table 2 and Figure 1.

Table 2. MAE and RMSE of Conversion Coefficient K of Every Station from 2016 to 2019

\begin{tabular}{cccccc}
\hline Station & $\begin{array}{c}\text { Latitu- } \\
\text { de }\left({ }^{\circ}\right)\end{array}$ & $\begin{array}{c}\text { Altitu- } \\
\text { de }(\mathrm{m})\end{array}$ & $\begin{array}{c}\text { Elevation } \\
\text { difference } \\
(\mathrm{m})\end{array}$ & $\begin{array}{c}\text { MAE } \\
/ 10^{-3}\end{array}$ & $\begin{array}{c}\text { RMSE } \\
/ 10^{-3}\end{array}$ \\
\hline Yinchun & 38.48 & 1112 & 255 & 2.261 & 2.790 \\
Minqin & 38.63 & 1367 & & 2.136 & 2.687 \\
\hline Pingliang & 35.55 & 1348 & \multirow{2}{*}{527} & 1.860 & 2.373 \\
Yuzhong & 35.87 & 1875 & & 2.011 & 2.497 \\
\hline Nanning & 22.63 & 126 & \multirow{2}{*}{1177} & 3.431 & 4.280 \\
Simao & 22.76 & 1303 & & 3.147 & 3.611 \\
\hline Yan'an & 36.60 & 959 & \multirow{2}{*}{1850} & 2.284 & 2.825 \\
Golmud & 36.41 & 2809 & & 4.216 & 4.637 \\
\hline Hanzhong & 33.06 & 509 & \multirow{2}{*}{3173} & 2.703 & 3.327 \\
Yushu & 33.01 & 3682 & & 5.841 & 6.072 \\
\hline Ganzi & 31.61 & 522 & \multirow{2}{*}{3986} & 3.270 & 3.651 \\
Naqu & 31.48 & 4508 & & 7.902 & 8.456 \\
\hline
\end{tabular}

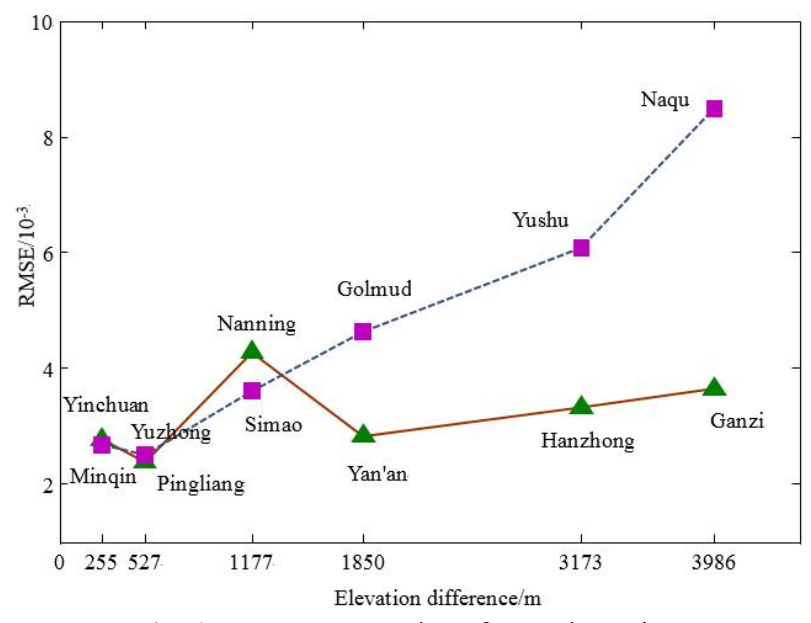

Fig. 1. RMSE Comparison for Each Station

According to Table 2 and Figure 1, the K-value models of Yinchuan \& Minqin and Pingliang \& Yuzhong with small altitude difference assumed similar accuracy and inconspicuous deviation. With the increase of altitude difference, the accuracy of K-value model in each station demonstrated a significant difference, and the accuracies of models in stations with higher altitude were lower. At the height difference of $1850 \mathrm{~m}$ (i.e. Golmud \& Yan'an), there was a notable accuracy 
difference of the K-value model between the two stations, as evidenced by the fact that the model accuracy of Yan'an station at $959 \mathrm{~m}$ was twice higher than that of Golmud station at $2809 \mathrm{~m}$. At the altitude difference of above $1850 \mathrm{~m}$, the accuracy difference of the model between the two stations gradually increased and the accuracy difference of the model with a greater altitude was about twice that of the model with a smaller altitude. At similar latitudes, the accuracy of K-value model was lower for stations with obvious topographic relief and greater altitude, which was significantly higher for stations with flat terrain and small altitude.

\section{Feasibility Analysis of PWV Inversion}

For the purpose of verifying the feasibility of inverting PWV using the H-model, eight sounding stations in the western area, namely Lhasa, Naqu, Changdu, Yushu, Xining, Dulan, Golmud and Hezuo, were selected as the research objects. Sounding data was introduced to directly calculate the Zenith wet delay (ZWD) in 2019. According to the formula $P W V=K^{*} Z W D$, the annual PWV of eight sounding stations in 2019 was obtained, the deviation between which and the PWV provided by the sounding stations was as shown in Figure 2. The distribution of the monthly mean PWV inverted by the $\mathrm{K}$-value model was as shown in Figure 3. The proportion of PWV at each station across the four seasons in the annual PWV was as shown in Figure 4.
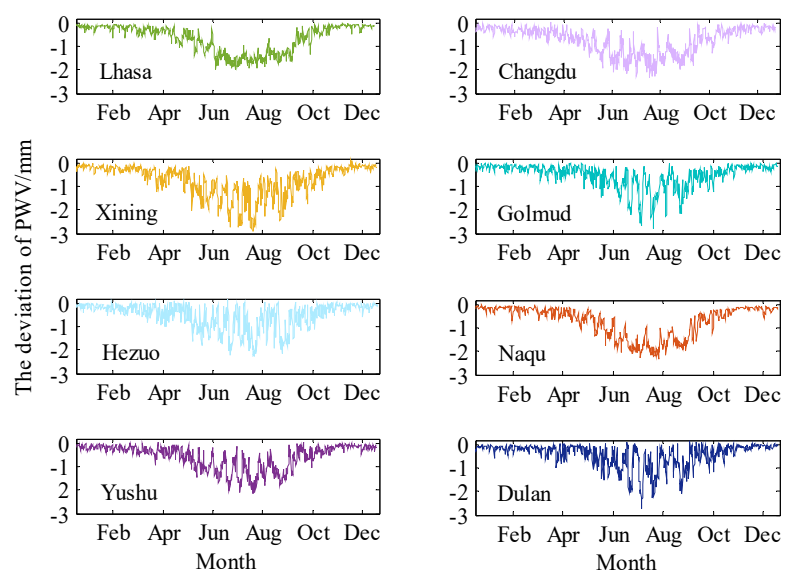

Fig. 2. Deviation between PWV Value Estimated by Combining Sounding Data and K-value Model and PWV Value Provided by Sounding Stations

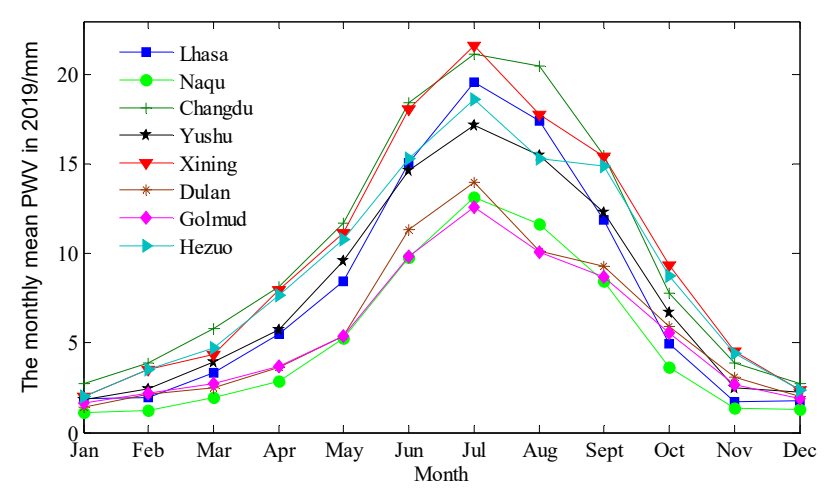

Fig. 3. Monthly Mean PWV Changes of Stations in 2019

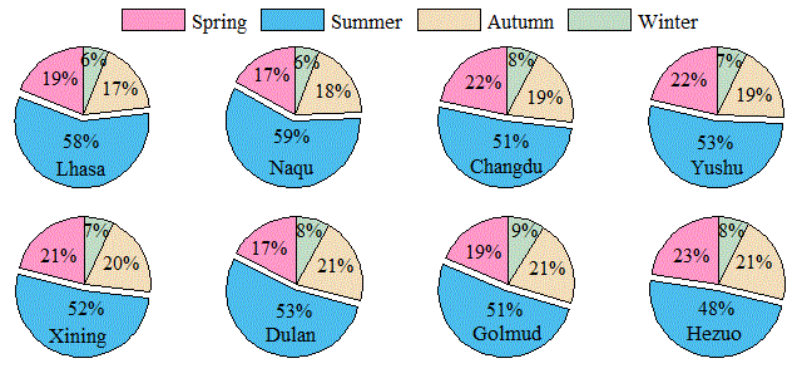

Fig. 4. Pie Chart of PWV's Seasonal Distribution Characteristics

As shown in Figure 2, there was a deviation of 0$\pm 3 \mathrm{~mm}$ between the PWV value obtained based on ZWD calculated by the sounding data in combination with the inversion of K-value model and that provided by sounding stations. As can be concluded from Figure 3, PWV was positively correlated with the distribution of its deviation, i.e., the larger the monthly average PWV, the greater the deviation. The maximum deviation occurred in summer when maximum precipitation took place. The monthly mean PWV values of each station were generally consistent over time and demonstrated seasonal characteristics. Specifically, PWV rose slowly in spring and peaked in the summer of July, followed by a gradual decline in autumn and reached the lowest value in winter. As shown in Figure 4, except for Hezuo, where the summer PWV accounted for $48 \%$ of whole year, the summer PWV of all other stations accounted for more than $50 \%$ of the whole year. Thus water vapor in the area mainly occurred in summer, suggesting a highly concentrated seasonal distribution of precipitation.

\section{Conclusion}

Based on the sounding data of 43 sounding stations in western China from 2016 to 2019, a K-value model was established in this paper to explore the applicability of the model. Stations varying in altitude were introduced to identify the impact of altitude difference on model accuracy. The PWV of eight sounding stations in 2019 was subsequently inverted using the $\mathrm{K}$-value model to verify its usability. The following conclusions were drawn:

(1) The establishment of regional models covering a large area improves the model's accuracy, and the Kvalue model assumes a higher accuracy in areas with flatter terrains.

(2) There was a $<3 \mathrm{~mm}$ deviation between the PWV inverted using the K-value model and the PWV provided by the sounding stations; the deviation is positively correlated with the PWV inverted using the K-value model.

(3) According to the PWV distribution inverted using the $\mathrm{K}$-value model, $\mathrm{PWV}$ in summer accounts for more than $50 \%$ of the whole year; water vapor is also more concentrated in summer, which suggests a highly concentrated seasonal distribution of precipitation. 


\section{Acknowledgement}

Guangxi Natural Science Foundation (2018GXNSFAA138168); science and technology projects of Guangxi surveying and Mapping Bureau (2016-E-01; 2018-E-01); science and technology projects of Guangxi Normal University (0819-2016L11).

\section{References}

1. J. G. Qu, OHIT, Calculation and Analysis of Transformation Coefficient K Value of Calculating Integrated Water Vapor, 3,35-37(2002)

2. T. R. Emardson, G. Elgered, JGR, Three Months of Continuous Monitoring of Atmospheric Water Vapor with a Network of Global Positioning System Receivers, D2, 1807-1820(1998)

3. T. R. Emardson, H. J. P. Derks, MP, On the Relation between the Wet Delay and the Integrated Precipitable Water Vapour in the European Atmosphere, 1, 61-68(2000):

4. L. Liu, X. Chen, H. Fei, JGG, Analysis of the Applicability of the Emardson Model for Transformation Coefficient of Water Vapor in the Region of Xinjiang, 50, 434-437(2016)

5. L.Liu, X. Chen, J. Li. Research on the model of atmospheric water vapor conversion factor with considering terrain fluctuation. ICIEOA, 98081M(2015)

6. X. Chen, Y. Yang, JGUT, The Applicability of H Model to Calculate the PWV in the Tibetan Plateau Area, 2, 283-288(2018) 